\title{
The Strategy of Power Demand Side Management Based on Energy Storage Technology Ying Chen
}

Department of Economic Management, North China Electric Power University, Baoding, China

Keywords: TOU, Load shifting, Vanadium redox flow battery, Premature convergence

Abstract. Aiming at the incompleteness of TOU for load shifting, we put forward the management strategy model of power demand side based on energy storage technology. And this paper use the particle swarm optimization algorithm to solve the model. The algorithm is tend to be premature convergence in dealing with high-dimensional problems. So we improve the algorithm .The test results show that the improved algorithm is better. Finally, through a certain area examples it simulate actual system quantitatively. The results verificate the validity of the model and controlling strategy.

\section{Introduction}

Load shifting in China is still more severe than the developed countries .In order to improve the effectiveness of the DSM, scientific research institutions focus more and more on energy storage technology.

The literature [1] introduces the forecasting system of wind power plants with vanadium redox flow battery. The literature [2] presents efficient large-scale storage technology including water pumping energy storage technology. The literature [3] introduces how to achieve stable voltage using super capacitor for fastly charging and discharging. The study focuses on the application of energy storage technology in grid connected with renewable energy, lacking of deep research on the application of technology in load shifting. In this paper, we take the vanadium redox flow energy storage technology as an example and establish the energy storage system model in load shifting.

\section{Power demand side management model based on energy storage technology}

Vanadium redox flow storage technology can effectively reduce the difference between peak and valley; We take the minimum of $\mathrm{Z}$ as target, the target $\mathrm{Z}$ is:

$$
\min Z=\min \sum_{j=t_{1}}^{t_{2}}\left(P_{Y j}-\sum_{i=1}^{M} P_{i j}-\frac{P_{Y j}-\sum_{i=1}^{M} P_{i j}}{t_{2}-t_{1}+1}\right)^{2}
$$

Where $t_{1}=$ the initial period of optimization of electricity; ${ }_{2}=$ the termination period of optimization of electricity ; ${ }^{P_{j}}=$ average load power for period ${ }^{j} ; M=$ the number of power storage stationr; $P_{i j}=$ charging or discharging load for the storage system ${ }^{i}$ for period $j$, a positive value indicates a discharge, a negative value indicates a charge;

In the power optimization process, we take a maximum of the annual cost of saving load minused by annual cost of storing load as target $\mathrm{W}$, the model $\mathrm{W}$ is:

$$
\max W=r\left(\sum_{i=1}^{M}\left|P_{i j}\right| / 2\right)\left(P_{f}-P_{g}\right)-C_{f}-C_{v}
$$

Where $P_{f}=$ price of peak period ; $P_{g}=$ price of valley period ; $C_{f}=$ the average fixed cost; $C_{v}=$ the average variable cost; $r=$ the energy conversion efficiency for each station; $Q=$ the total load absorbed by the energy storage system.

The actual charging and discharging power do not exceed the limited scope : 


$$
\max \left(-P_{f \max },-P_{s \max }\right) \leq P_{i j} \leq \min \left(P_{f \max }, P_{s \max }\right)
$$

Where $P_{f \max }=$ the maximum of allowable power for the grid; $P_{s \max }=$ the maximum of allowable power for the energy storage device.

In power optimization process, keep the users' total demand unchanged:

$$
\sum_{j=t_{1}}^{t_{2}} \sum_{i=1}^{M} P_{i j}=\sum_{j=t_{1}}^{t_{2}} P_{Y j}
$$

\section{Vanadium redox flow battery}

According to $\Delta U=(P R+Q X) / U$, considering the active influence on system to adjust the voltage, we add negative feedforward compensation and formula after changed to calculate the active power :

$$
P_{\text {set }}=\left(K_{w p}+K_{w i}\right) / \Delta \eta
$$

Where $K_{w p}, K_{w i}=$ scaling factor and integration factor for frequency - active PI controller; $P_{\text {set }}=$ the active power absorbed by energy storage system; $\Delta \eta=$ the rate of resistance due to change of power system ;

The control equation is:

$$
\begin{aligned}
& U_{q}=-\left(K_{i p}+K_{i I}\right)\left(i_{q}^{*}-i_{q}\right)-L_{i d} \\
& U_{d}=-\left(K_{i p}+K_{i I}\right)\left(i_{d}^{*}-i_{d}\right)+L_{i q}
\end{aligned}
$$

Where $K_{i p} 、 K_{i I}=$ the proportional control gain and integral control gain for PI control ; $i_{q}^{*} 、 i_{d}^{*}=$ the current flowing into the vector in the component $q, d$ shaft; $i_{q} i_{d}=$ the command value; $L_{i q} 、 L_{i d}=$ the scale factor for frequency in the $q, d$ shaft.

Active power conversion for AC side and grid side is:

$$
r=P / P_{\text {set }}
$$

Where $r=$ the conversion efficiency of the active energy.

\section{Solving process based on the improved PSO algorithm}

The literature [4] points out that one of rules for birds is matching with near peer. Accordingly, the steps of improved HSPO algorithm are as follows:

Step 1: Initialize parameter value, randomly generate all the positions and velocities of all particles;

Step 2: According to the established objective function, calculate the fitness value of particles;

Step3: "speed" detection. When the number of iterations to complete the $1 / 3$ of iteration times, detect the possibility of mismatch of speed;

Step 4: Speed control. Adjust the inertia weight according to the formula (9):

$$
\omega=\omega_{\max }-\left(\omega_{\max }-\omega_{\min }\right) \frac{k}{\beta-k}
$$

Where $\omega_{\max } \vee \omega_{\min }=$ the maximum, minimum value of inertia weight; $k=$ the iteration number for mismatch of speed; $\beta=$ the eviation of the coefficient;

Step 5: Update the velocity and position of particle;

Step 6: Computate the fitness of each particle, update the optimal position of the group;

Step7: Judge whether the termination condition is reached and determine to stop operation, or continue to perform.

\section{Example analysis}

Using the data of a typical day in a certain region, we simulate the scenarios. At the same time 
the power factor of equipment is more than 0.95 , the line resistance about $60 \Omega$.

The type of energy storage station is the $5 \mathrm{MW} / 10 \mathrm{MWh}$ of vanadium redox flow battery. The energy conversion rate is $81.2 \%$. The working voltage ranges from $400 \mathrm{DC}$ to $620 \mathrm{~V}$. The flow battery consists of 1 sets of $500 \mathrm{~kW}$ PCS and 2 sets of $250 \mathrm{~kW}$ PCS to control the charging and discharging. The maintenance cost is $0.009 \mathrm{RMB} / \mathrm{kwh}$. Assuming that the device can be used for 15 years, the cost is 0.75 yuan.The average cost of purchasing electricity is 0.67 yuan. According to the formula (8), set the energy conversion efficiency as $78.824 \%$.
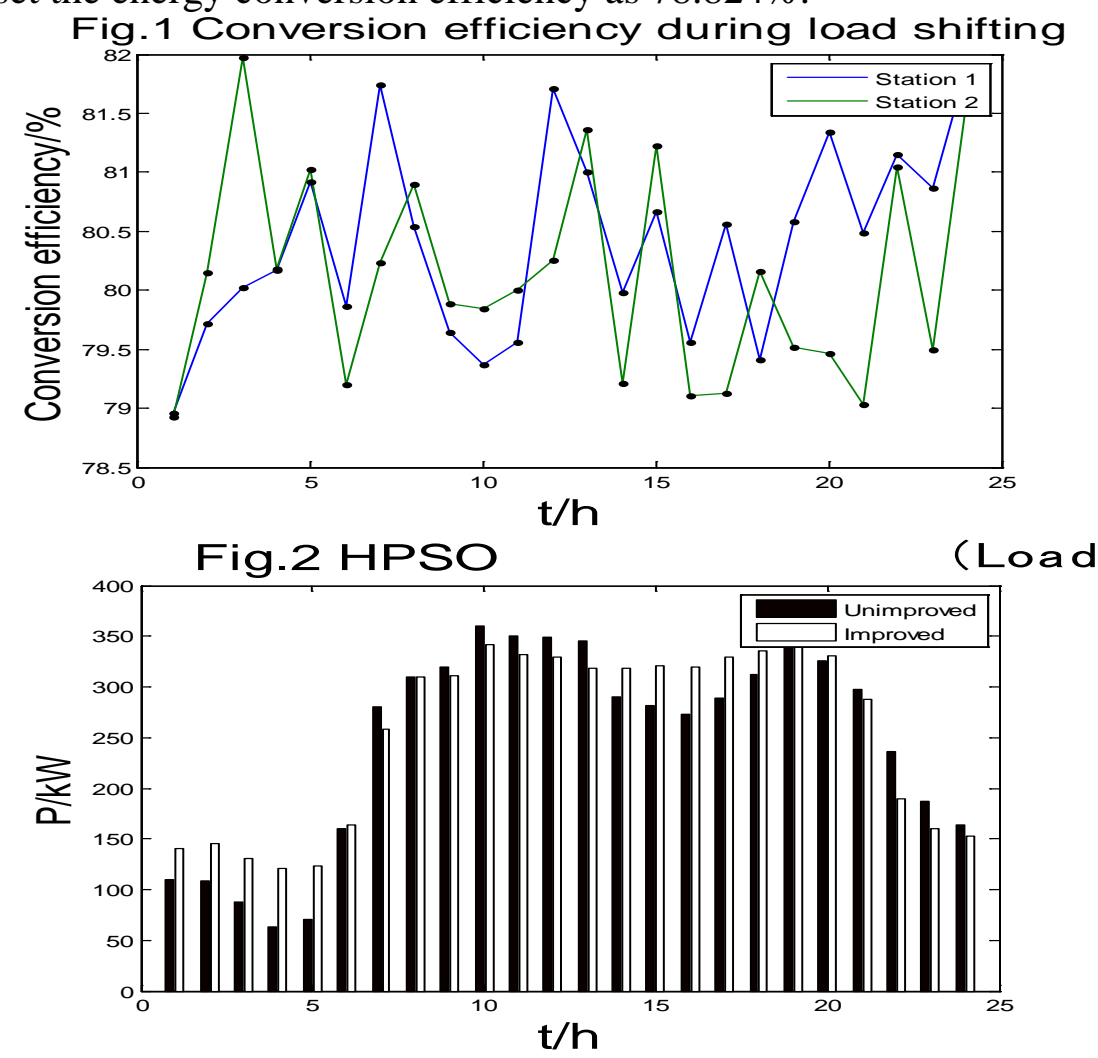

Fig.1 shows the curve the conversion efficiency of power station after improved .When they are complied with the preset energy conversion efficiency value, the model can save the costs of consumers for electricity. Fig.2 and fig.3 show the effect of 2 storage stations simultaneously taking part in load shifting. Considering the load shifting time,before adjusted, load rate is 0.525 ; After the regulation,load rate is 0.585 , The daily load rate obtained by the two algorithms are 0.697 and 0.683. After adjusted, the difference of load between peak and valley decreases and the load curve is more smooth. Fig. 4 shows the curves of fitness value based on two kinds of adaptive algorithms and indicates that the improved algorithm is better.

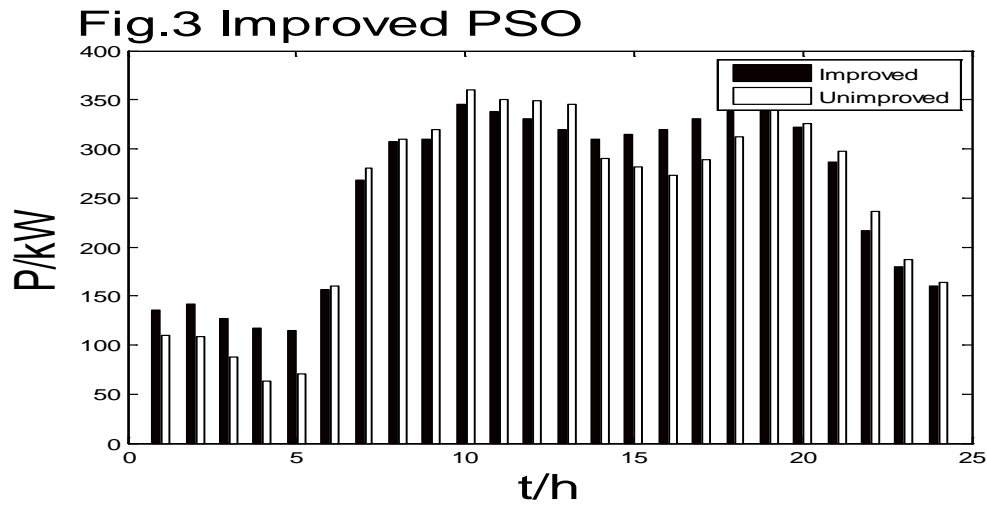

( $\mathbf{L}$ 


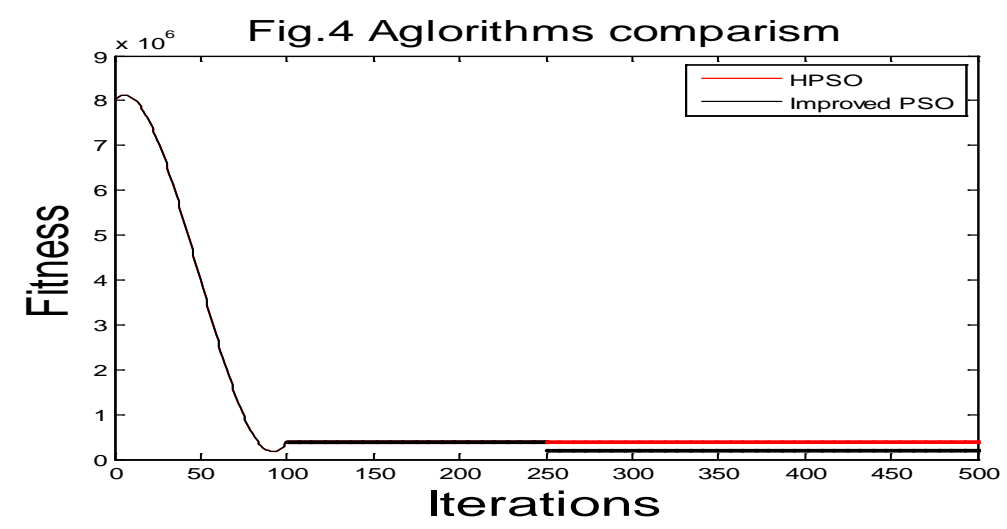

\section{Conclusions}

The control strategy meets the demand of the power system, energy storage system,and the users based on the improved PSO algorithm for the energy storage.Numerical results show that the control strategy can achieve better for the load shifting and suppress the fluctuation in the power generation. In addition, the energy storage system used to controlling strategies in DSM is not immutable and frozen so should excavate factors which influence power system electrical efficiency to save energy and reduce emission better. This paper aims at the research on the controlling strategy of energy storage in terms of technology and economy, and provide the theoretical support for the practical application .

\section{References}

[1]Zonghao Liu ,Huamin Zhang,Sujun Gao, Xiangkun Ma,Yufeng Liu. Wind Field matching with the World's Largest Vanadium Redox Flow Battery Energy Storage System [J]. Storage Science and Technology, 2014, (3).

[2]Tingjie Guo. The Overview of Foreign New Battery Development [J]. Energy-saving Technology, 1994, (1): 9-14

[3]Yunling Wang ,Jie Ceng, Buhan Zhang. Dynamic Pressure Regulator Based on Power Capacitor Energy Storage System [J]. Power System Technology, 2007, 31 (8): 58-62

[4]ReynoldC.W. A Distributed Behavioral Model[J]. Computer Graphics, 1987,24 (4): 25-34 